\title{
Quantitative Structure-Activity Relationships and Mixture Toxicity Studies of Chloro- and Alkylanilines at an Acute Lethal Toxicity Level to the Guppy (Poecilia reticulata)
}

\author{
Joop Hermens, Peter Leeuwangh, and Aalt Musch \\ Department of Veterinary Pharmacology, Pharmacy and Toxicology, University of Utrecht, \\ Biltstraat 172, 3572 BP Utrecht, The Netherlands
}

Received August 2, 1983

\begin{abstract}
Quantitative structure-activity relationships (QSARs) were calculated for the acute lethal toxicities (14-day $\mathrm{LC}_{50}$ ) to the guppy (Poecilia reticulata) of anilines, with several hydrophobicity characteristics and the Hammett $\sigma$ constant as parameters. Calculated and experimental $P_{\text {oct }}$ values and $\Pi$ constants were used as hydrophobicity characteristics. Introduction of Hammett constants improved the quality of the QSARs. Together with the QSAR studies the toxicities of mixtures of the anilines were determined. The toxicities of three mixtures did not deviate from concentration addition.
\end{abstract}

\section{INTRODUCTION}

For the past few years quantitative structure-activity relationships (QSAR), already common in pharmacology, have also been used in aquatic toxicity studies (Veith and Konasewich, 1975; Könemann, 1980; Hansch, 1978). The classical empirical equation of Hansch and Fujita (1964) to describe relationships between biological activity and chemical structure is given in

$$
\log \frac{1}{c}=k_{1}(\log P)^{2}+k_{2}(\log P)+k_{3} \sigma+k_{4} E_{\mathrm{s}}+k_{5}
$$

where

$c=$ molar concentration, characteristic for a certain activity, i.e., $\mathrm{LC}_{50}$;

$P=$ partition coefficient, usually between $n$-octanol and water $\left(P_{\mathrm{oct}}\right)$, a characteristic of hydrophobic properties;

$\sigma=$ Hammett constant, a characteristic of electronic effects;

$E_{\mathrm{s}}=$ Taft constant, a characteristic of steric effects;

$k_{i}=$ constants obtained by fitting the equation to experimental data.

As an alternative characteristic of hydrophobic properties $\Pi$ constants can be used. The advantage of using $P_{\mathrm{oct}}$ is that the toxicities of chemicals with nonrelated structures can be compared, because $P_{\text {oct }}$ represents the hydrophobic properties of a whole molecule. These $P_{\text {oct }}$ values can be determined experimentally or, more easily, can be calculated using the "hydrophobic fragmental constant" method of Rekker (1977). II constants, on the other hand, can be used only within a set of structure-related chemicals. The constant $\Pi$ of a substituent $X$ is defined as (Fujita et al., 1964)

$$
\Pi_{X}=\log P_{\mathrm{R} X}-\log P_{\mathrm{RH}}
$$


where

$$
\begin{aligned}
\Pi_{X} & =\text { substituent constant; } \\
P_{\mathrm{R} X} & =P_{\mathrm{oct}} \text { of a derivative; } \\
P_{\mathrm{RH}} & =P_{\mathrm{oct}} \text { of parent molecule. }
\end{aligned}
$$

When two substituents are placed on an aromatic ring the electronic effects of one upon the other can change the $\Pi$ value of each. With anilines, for instance, the hydrophobicity of a derivative of aniline is influenced by the electronic character of the substituent(s) (electron withdrawing or releasing). The advantage of the $\Pi$ system is that more sets of $\Pi$ values are tabulated for systems in which such interacting groups are present or absent (Fujita et al., 1964; Norrington et al., 1975; Hansch and Leo, 1979). For example, in the case of compounds with electron-donating side chains, the partition coefficient is better described using $\Pi^{-}$values (Norrington et al., 1975).

Together with hydrophobocity, electronic distribution in a molecule can also influence its activity. The most useful electronic structural parameter is the Hammett $\sigma$ constant. This constant is defined as (Johnson, 1973).

where

$$
\sigma=\log K_{\mathrm{R} X}-\log K_{\mathrm{RH}}
$$

$K_{\mathrm{RX}}=$ ionization constant of meta or para derivative of benzoic acid;

$K_{\mathrm{RH}}=$ ionization constant of benzoic acid.

This equation is not applicable to ortho substituents due to steric effects on the reaction center. Apparent $\sigma$ constants, however, have been tabulated by Clark and Perrin (1964) for ortho substituents in anilines. These values were obtained from $\mathrm{p} K_{a}$ determinations.

In this study we calculated correlations between the acute lethal toxicity to the guppy of a series of anilines (chloro- and alkylanilines) with different characteristics of hydrophobicity [ $\log P_{\text {oct }}$ calculated after Rekker (1977), $\Pi$ constants, and experimental $\log P_{\text {oct }}$ values] together with Hammett $\sigma$ constants. Hammett constants were used as parameters, because we expected the electronic character of the lone electron pair on the $\mathrm{N}$ atom to influence the toxicity of the anilines.

Using equitoxic concentrations based on the $\mathrm{LC}_{50}$ values of single compounds we also tested the $50 \%$ toxicity level of mixtures of the anilines. Similar studies were performed by Könemann (1981a) with mixtures of chlorophenols and anesthetic agents. We used the mixture toxicity index (MTI) of Könemann (1981a) to interpret the results of the mixture toxicity experiments. This MTI, developed to compare the toxicities of mixtures of more than two chemicals, equals 1 in the case of concentration addition.

\section{METHODS}

Experiments to determine the 14-day $\mathrm{LC}_{50}$ to the guppy (Poecilia reticulata) were conducted as described earlier by Könemann (1981b). Stock solutions of the anilines were prepared in 2-propanol, instead of acetone, because gas chromatographic analyses showed interaction between the anilines and acetone (possibly forming a Schiff base). Also, $\mathrm{LC}_{50}$ determinations with acetone as solvent were not reproducible, especially in experiments with alkylanilines. 
The substituent constants used in the correlation analyses are summarized in Table 1. $\Pi^{-}$constants were taken because they are appropriate for anilines. These substituent constants from Table 1 are additive; thus the parameter values of polysubstituted anilines can be obtained by adding the individual constants for each substituent.

QSARs were calculated with a computer program, based on the method of least squares. The significance of the improvements of the QSARs was tested with an $F$ test as described by Hansch (1973).

Experiments with mixtures were carried out under the same conditions used for the experiments with separate chemicals. All mixtures were prepared in equitoxic concentrations (identical fractions of the $\mathrm{LC}_{50}$ ).

\section{RESULTS AND DISCUSSION}

\section{QSAR Studies}

The results of the $\mathrm{LC}_{50}$ determinations, together with the hydrophobicity and electronic characteristics, are summarized in Table 2. The QSARs calculated with aniline and chloroanilines are summarized in Table 3 [Eqs. (4)-(11)]. The correlations between hydrophobicity characteristics and Hammett constants are given in Eqs. (12)-(14) (also in Table 3).

Introduction of a $\left(\log P_{\text {oct }}\right)^{2}$ term [Eq. (5)] hardly seems to improve the quality of the QSAR as would be expected since in the $\log P_{\text {oct }}$ range of 1.0-3.9 a nonlinear relationship between 14-day $\mathrm{LC}_{50}$ and $\log P_{\text {oct }}$ is unlikely. In experiments with the same toxicity criterion with another group of organic chemicals by Könemann (1981b) no deviations from linearity were found at $\log P_{\text {oct }}$ up to 5.7.

Introduction, however, of the Hammett constant improves the quality of the correlations (statistically significant at $P<0.05$ ) in all three cases [Eqs. (6), (8), (10)]. These correlations, however, can be affected by the rather high correlations between hydrophobicity characteristics and Hammett constants [Eqs. (12)-(14)]. The correlation between $\Pi^{-}$and $\sigma$ [Eq. (14)] is the lowest one, while in the QSAR with $\Pi^{-}$introduction

TABLE 1

SUBSTITUENT CONSTANTS

\begin{tabular}{llll}
\hline Aniline & $f_{\text {oct }}{ }^{a}$ & $\Pi^{-b}$ & $\sigma^{c}$ \\
\hline $\mathrm{H}$ & 0.175 & 0 & 0 \\
$o-\mathrm{Cl}$ & 0.922 & 0.69 & 0.67 \\
$m-\mathrm{Cl}$ & 0.922 & 1.04 & 0.37 \\
$p-\mathrm{Cl}$ & 0.922 & 0.93 & 0.23 \\
$o-\mathrm{CH}_{3}$ & 0.702 & 0.49 & 0.10 \\
$m-\mathrm{CH}_{3}$ & 0.702 & 0.50 & -0.07 \\
$p-\mathrm{CH}_{3}$ & 0.702 & 0.48 & -0.17 \\
$o-C_{2} \mathrm{H}_{5}$ & 1.232 & 0.99 & 0.05 \\
$m-\mathrm{C}_{2} \mathrm{H}_{5}$ & 1.232 & 0.99 & -0.07 \\
$p-\mathrm{C}_{2} \mathrm{H}_{5}$ & 1.232 & 0.98 & -0.15 \\
\hline
\end{tabular}

a Hydrophobic fragmental constants from Rekker (1977).

${ }^{b}$ Data drawn from Norrington et al. (1975).

${ }^{c}$ Data drawn from Clark and Perrin (1964). 
TABLE 2

LC $_{50}$ VALUES AND HYDROPHOBICITY AND ELECTRONIC CHARACTERISTICS OF THE ANILINES

\begin{tabular}{lccccc}
\hline \multicolumn{1}{c}{ Aniline } & $\begin{array}{c}\text { Log } P_{\text {oct }}{ }^{a} \\
\text { (calc.) }\end{array}$ & $\begin{array}{c}\text { Log } P_{\text {oct }}{ }^{b} \\
(\text { exp.) }\end{array}$ & $\Sigma \Pi^{-c}$ & $\Sigma \sigma^{c}$ & Log LC ${ }^{c}{ }^{d}$ \\
\hline $\mathrm{H}$ & 1.03 & 0.90 & 0.00 & 0.00 & 3.13 \\
$2-\mathrm{Cl}$ & 1.76 & 1.99 & 0.69 & 0.67 & 1.69 \\
$3-\mathrm{Cl}$ & 1.76 & 2.00 & 1.04 & 0.37 & 2.02 \\
$4-\mathrm{Cl}$ & 1.76 & 2.02 & 0.93 & 0.23 & 2.31 \\
$2,5-\mathrm{di}-\mathrm{Cl}$ & 2.42 & 2.75 & 1.73 & 1.04 & 1.01 \\
$2,4-\mathrm{di}-\mathrm{Cl}$ & 2.42 & 2.78 & 1.62 & 0.90 & 1.59 \\
$3,5-$ di-Cl & 2.42 & 2.90 & 2.08 & 0.74 & 1.38 \\
$3,4-\mathrm{di}-\mathrm{Cl}$ & 2.42 & 2.67 & 1.97 & 0.60 & 1.59 \\
$2,3,4-$ tri-Cl & 3.17 & 3.33 & 2.66 & 1.27 & 0.85 \\
$2,4,5-$ tri-Cl & 3.17 & 3.45 & 2.66 & 1.27 & 1.00 \\
$2,3,4,5-$ tetra-Cl & 3.92 & 4.04 & 3.70 & 1.64 & 0.19 \\
$2-\mathrm{CH}_{3}$ & 1.54 & - & 0.49 & 0.10 & 2.88 \\
$3-\mathrm{CH}_{3}$ & 1.54 & - & 0.50 & -0.07 & 2.53 \\
$4-\mathrm{CH}_{3}$ & 1.54 & - & 0.48 & -0.17 & 2.00 \\
$2-\mathrm{C}_{2} \mathrm{H}_{5}$ & 2.07 & - & 0.99 & 0.05 & 2.79 \\
$3-\mathrm{C}_{2} \mathrm{H}_{5}$ & 2.07 & - & 0.99 & -0.07 & 2.35 \\
$4-\mathrm{C}_{2} \mathrm{H}_{5}$ & 2.07 & - & 0.98 & -0.15 & 2.38 \\
\hline
\end{tabular}

${ }^{a}$ Calculated after Rekker (1977) with substituent constants from Table 1 and with $f_{\mathrm{C}_{6} \mathrm{Hs}_{\mathrm{s}}}=1.886, f_{\mathrm{C}_{6} \mathrm{H}_{4}}$ $=1.688, f_{\mathrm{C}_{6} \mathrm{H}_{3}}=1.431, f_{\mathrm{C}_{6} \mathrm{H}_{2}}=1.256, f_{\mathrm{C}_{6 \mathrm{H}}}=1.081$.

${ }^{b}$ Values obtained with RP-HPLC technique; data drawn from Hammers et al. (1982).

${ }^{c}$ Calculated with constants from Table 1.

${ }^{d}$ 14-day $\mathrm{LC}_{50}$ to guppy; $\mathrm{LC}_{50}$ in $\mu \mathrm{mol} /$ liter.

of the Hammett $\sigma$ gives a significant improvement at $P<0.01$. This, together with the fact that introduction of $\sigma$ in all cases improves the quality of the QSARs, although not all these improvements are statistically significant at $P<0.01$, is suggestive of a

TABLE 3

QSARs WITH ANILINE AND CHLORANILINES

\begin{tabular}{cccc}
\hline & \multicolumn{2}{c}{ QSAR, $n=11$ (first 11 compounds from Table 2) } \\
\cline { 2 - 4 } Eq. & \multicolumn{1}{c}{$\log 1 / \mathrm{LC} C_{50}=$} & $r^{a}$ & $s^{b}$ \\
\hline$(4)$ & $0.922 \log P_{\text {calc. }}-3.72$ & 0.946 & 0.27 \\
$(5)$ & $-0.150\left(\log P_{\text {calc. }}\right)^{2}+1.67 \log P_{\text {calc. }}-4.56$ & - & 0.26 \\
$(6)$ & $0.245 \log P_{\text {calc. }}+1.17 \Sigma \sigma-3.04$ & 0.970 & 0.22 \\
$(7)$ & $0.881 \log P_{\text {exp. }}-3.83$ & 0.959 & 0.24 \\
$(8)$ & $0.387 \log P_{\text {exp. }}+0.917 \Sigma \sigma-3.27$ & 0.977 & 0.19 \\
$(9)$ & $0.701 \Sigma \Pi^{-}-2.74$ & 0.928 & 0.31 \\
$(10)$ & $0.200 \Sigma \Pi^{-}+1.17 \Sigma \sigma-2.80$ & 0.973 & 0.20 \\
$(11)$ & $1.56 \Sigma \sigma-2.76$ & 0.967 & 0.21 \\
$(12)$ & $\log P_{\text {calc. }}=1.58 \Sigma \sigma+1.14$ & 0.955 & 0.25 \\
$(13)$ & $\log P_{\text {exp. }}=1.65 \Sigma \sigma+1.31$ & 0.943 & 0.30 \\
$(14)$ & $\Sigma \Pi^{-}=1.95 \Sigma \sigma+0.19$ & 0.914 & 0.45 \\
\hline
\end{tabular}

${ }^{a}$ Correlation coefficient.

${ }^{b}$ Standard error of estimate. 
real influence of electronic properties in the molecule on the toxicity of the chloroanilines.

A reduced correlation between hydrophobicity and Hammett constants may result in more distinct contributions of each of the molecular properties and therefore in a more pronounced improvement of the QSARs. In order to reduce the correlations between the hydrophobicity terms and Hammett constants we extended the group of chloroanilines with 6 alkylanilines. The calculated QSARs with aniline, 10 chloroanilines, and 6 alkylanilines are given in Table 4.

The correlation between toxicity and $\log P_{\text {calc. }}$ together with $\sigma$ [Eq. (16)] has a significantly smaller $(P<0.01)$ standard error compared with the QSAR with $\log P$ alone [Eq. (15)]. This and Eq. (10) are the only examples in which the improvements obtained by introduction of the Hammett constants are statistically significant at $P$ $<0.01$. This is probably related to the relatively low correlations between hydrophobicity characteristics and Hammett constants in Eqs. (14) and (20).

Although introduction of Hammett constants improves the QSARs for the 17 anilines [Eq. (16), (18)] the equations remain less satisfactory than the equations for the 11 chloroanilines [Eqs. (6), (8), (10)]. It is concluded that other characteristics must influence the toxicity or that the alkylanilines act differently from the chloroanilines.

It is not clear what influence the Hammett constant represents in the QSARs we calculated. The influence of $\mathrm{p} K_{a}$, which is directly related to the Hammett constant, on the bioactivation of amines to their $\mathrm{N}$-hydroxy derivatives has been observed (Gorrod, 1978). Also, the influence of Hammett constants on the enzymatic acylation of anilines is known (Hansch et al., 1965). The influence of Hammett constants cannot be explained by ionization of the anilines, because at the $\mathrm{pH}$ of the experiments $( \pm 7.3)$ as well as at physiological $\mathrm{pH}$, the tested anilines are less than $1 \%$ ionized. The highest $\mathrm{p} K_{a}$ value is that of 4-methylaniline which has a $\mathrm{p} K_{a}$ of 5.08 (Perrin, 1965).

The anilines differ with respect to the influence of the Hammett constant from the nonreactive organic chemicals with unspecific toxicity (central nervous depression as with anesthetics). As shown in an earlier study by Könemann (1981b), the 14-day $\mathbf{L C}_{50}$ for the guppy of 50 such chemicals correlated perfectly with $P_{\text {oct }}$ as the only parameter. This QSAR (with an intercept of 4.87 ) probably predicts the minimum toxicity of nonreactive organic chemicals. In comparison with this group of nonreactive

TABLE 4

QSARS WITH ANILINE, CHLOROANILINES, AND ALKYLANILINES

\begin{tabular}{llcc}
\hline & \multicolumn{2}{c}{ QSAR, $n=17$ (all compounds from Table 2) } \\
\cline { 2 - 4 } Eq. & \multicolumn{1}{c}{$\log 1 / L C_{50}=$} & $r$ & $s$ \\
\hline 15 & $0.988 \log P_{\text {calc. }}-4.02$ & 0.885 & 0.39 \\
16 & $0.407 \log P_{\text {calc. }}+0.846 \Sigma \sigma-3.17$ & 0.935 & 0.31 \\
17 & $0.749 \Sigma \Pi^{-}-2.90$ & 0.906 & 0.35 \\
18 & $0.359 \Sigma \Pi^{-}+0.745 \Sigma \sigma-2.73$ & 0.937 & 0.30 \\
19 & $1.29 \Sigma \sigma-2.50$ & 0.917 & 0.33 \\
20 & $\log P_{\text {calc }}=1.09 \Sigma \sigma+1.64$ & 0.864 & 0.38 \\
21 & $\Sigma \Pi^{-}=1.52 \Sigma \sigma+0.63$ & 0.893 & 0.45 \\
\hline
\end{tabular}


chemicals the chloroanilines with a QSAR intercept of 3.72 [Eq. (4)] are about 10 times more toxic. The higher toxicity of the anilines can be explained in several ways. The toxicity of the anilines may be related to a more specific mode of action. Anilines, especially the $N$-hydroxy metabolites, are known as ferrihemoglobin-forming agents (Kiese, 1974). Perhaps the higher toxicity is due to the reactivity of the $\mathrm{NH}_{2}$ group toward carbonyl groups in macromolecules. From our study, however, with mortality as the overall criterion of effect, it is not possible to indicate which mode of action(s) is responsible for the toxicity of the anilines.

\section{Mixture Toxicity Studies}

The toxicities of three equitoxic mixtures were determined. The results of these experiments are given in Table 5. The joint toxicity of all three mixtures appears to be concentration additive (MTI 1.0). The good-quality QSARs for the anilines (especially the chloroanilines) indicate a similar mode of action. The toxicities of mixtures of chemicals with similar modes of action are expected to be concentration additive as was earlier found by Könemann (1981a) for nonreactive organic chemicals with a potency for unspecific depression of the central nervous system. From the results of mixture toxicity experiments alone, similar actions of chemicals cannot be concluded. For example, in a study by Hermens and Leeuwangh (1982), the joint toxicity of some of the mixtures composed of substances with diverse chemical structures and modes of action was also near concentration addition. Therefore the observation of concentration addition in the mixture of 17 chemicals (mixture 3 in Table 3 ) is not an argument in itself in support of a similarity of action of the chloroand alkylanilines.

\section{CONCLUSIONS}

The acute lethal toxicity (14-day LC50) of anilines to guppies is a function of the hydrophobic and electronic properties of these compounds because a QSAR with $P_{\text {oct }}$ and Hammett constants yielded a significantly better correlation than $P_{\text {oct }}$ alone.

TABLE 5

RESULTS OF MIXTURE TOXICITY EXPERIMENTS

\begin{tabular}{|c|c|c|c|}
\hline \multirow[b]{2}{*}{ Composition of mixture: } & \multicolumn{3}{|c|}{ Mixture } \\
\hline & $\frac{1}{6 \text { alkyl anilines }}$ & $\begin{array}{l}2 \\
\text { aniline } \\
10 \text { chloroanilines }\end{array}$ & $\begin{array}{l}\quad 3 \\
\text { aniline } \\
6 \text { alkyl anilines } \\
10 \text { chloroanilines }\end{array}$ \\
\hline Number of chemicals $(n)$ & 6 & 11 & 17 \\
\hline$M^{a}$ & 1.1 & 1.1 & 1.1 \\
\hline $\mathrm{MTI}^{b}$ & 0.95 & 0.96 & 0.97 \\
\hline $\mathrm{SD}_{\mathrm{MTI}}{ }^{c}$ & 0.13 & 0.10 & 0.08 \\
\hline
\end{tabular}

\footnotetext{
${ }^{a} M=$ sum of the concentrations, expressed as equal fractions of the $\mathrm{LC}_{50}$ of each component.

${ }^{b}$ MTI calculated after Könemann (1981a).

${ }^{c} \mathrm{SD}_{\mathrm{MTI}}$ : standard deviation in MTI calculated as described by Könemann (1981a), with an estimated error in $\log \mathrm{LC}_{50}$ of 0.10 .
} 
The three characteristics of hydrophobicity (calculated and experimental $\boldsymbol{P}_{\text {oct }}$ values and $\Pi^{-}$constants) result only in small differences in the calculated QSARs. Only the correlations between the three hydrophobicity characteristics and the Hammett constants influence the statistical reliability of the improvements of the QSARs with Hammett constants as parameter.

The toxicities of mixtures of the anilines corresponded to concentration addition.

\section{ACKNOWLEDGMENTS}

This work was supported by the Ministry of Health and Environmental Protection. We wish to thank Professor H. van Genderen and Dr. W. H. Könemann for their stimulating discussion. The authors are also indebted to Mr. F. J. M. Busser for his assistance in chemical analysis.

\section{REFERENCES}

Clark, J., AND Perrin, D. D. (1964). Prediction of the strengths of organic bases. Q. Rev. Chem. Soc. $18,295-320$.

FujITA, T., IWASA, J., AND HaNSCH, C. (1964). A new substituent constant П, derived from partition coefficients. J. Amer. Chem. Soc. 86, 5175-5180.

GORROD, J. W. (1978). The current status of the pKa concept in the differentiation of enzymic N-oxidation. In Biological Oxidation of Nitrogen (J. W. Gorrod, ed.), pp. 201-210. Elsevier, Amsterdam.

HAMmers, W. E., MeURS, G. J., AND DE LIGNY, C. L. (1982). Correlations between liquid chromatographic capacity ratio data on Lichrosorb RP-18 and partition coefficients in the octanol-water system. J. Chromatogr. 247, 1-13.

HANSCH, C. (1973). Quantitative approaches to pharmacological structure-activity relationships. In StructureActivity Relationships (C. J. Cavallito, ed.), Vol. 1, pp. 150-158. Pergamon, Oxford.

HANSCH, C. (1978). Recent advances in biochemical QSAR. In Correlation Analysis in Chemistry, Recent Advances (N.B. Chapman and J. Shorter, eds.), pp. 397-438. Plenum, New York.

HANSCH, C., DeutSCH, E. W., and SMITH, R. N. (1965). The use of substituent constants and regression analysis in the study of enzymic reaction mechanisms. J. Amer. Chem. Soc. 87, 2738-2742.

HANSCH, C., AND FUJITA, T. (1964), $\rho-\sigma-\pi$ Analysis. A method for the correlation of biological activity and chemical structure. J. Amer. Chem. Soc. 86, 1616-1626.

HansCh, C., AND LEO, A. J. (1979). Substituent Constants for Correlation Analysis in Chemistry and Biology. Wiley, New York.

HeRmens, J., AND LeEuWANGH, P. (1982). Joint toxicity of mixtures of 8 and 24 chemicals to guppy (Poecilia reticulata). Ecotoxicol. Environ. Safety 6, 302-310.

JoHnson, C. D. (1973). The Hammett Equation. Univ. Press, Cambridge.

KIESE, M. (1974). Methemoglobinemia: A Comprehensive Treatise. CRC Press, Cleveland, Ohio.

KÖNEMANN, H. (1980). Structure-activity relationships and additivity in fish toxicities of environmental pollutants. Ecotoxicol. Environ. Safety 4, 415-421.

KONEMANN, H. (1981a). Fish toxicity tests with mixtures of more than two chemicals: A proposal for a quantitative approach and experimental results. Toxicology 19, 229-238.

KÖNEMANN, H. (1981b). Quantitative structure-activity relationships in fish toxicity studies. 1. Relationship for 50 industrial pollutants. Toxicology 19, 209-221.

Norrington, F. E., HYDE, R. M., Williams, S. G., AND Wootton, R. (1975). Physiochemical-activity relations in practice. 1. A rational and self consistent data bank. J. Med. Chem. 18, 604-607.

Perrin, D. (1965). Dissociation Constants of Organic Bases in Aqueous Solutions, Compiled for IUPAC. Butterworths, London (first suppl. 1972).

REKKER, R. F. (1977). The Hydrophobic Fragmental Constant. Elsevier, Amsterdam.

VeITH, G. D., AND KONASEWICH, D. E. (eds.) (1975). Structure-Activity Correlations in Studies of Toxicity and Bioconcentration with Aquatic Organisms. Great Lakes Advisory Board, Windsor/Ontario. 